\title{
Effect of Pyridoxal Phosphate on Atherosclerosis and Nephropathy Progression in Atherosclerotic Rats
}

\author{
Sina Mahdavifard. ${ }^{*}$ (iD), Manochehr Nakhjavani ${ }^{2}$ if \\ 1. Dept. of Clinical Biochemistry, Faculty of Medical Sciences, Ardabil University of Medical Sciences, Ardabil, Iran \\ 2. Dept. of Endocrinology and Metabolism, Faculty of Medicine, Tehran University of Medical Sciences, Tehran, Iran
}

\begin{tabular}{c}
\hline Article Info \\
\hline doi 10.30699/jambs.29.132.21 \\
\hline Received: 2019/11/23; \\
Accepted: 2020/06/12; \\
Published Online: 01 Oct 2020 \\
\hline Use your device to scan and read the \\
article online \\
Q \\
\hline
\end{tabular}

Corresponding Information:

Sina Mahdavifard,

Dept. of Clinical Biochemistry, Faculty

of Medical Sciences, Ardabil

University of Medical Sciences,

Ardabil, Iran

E-Mail:

s.mahdavifards@yahoo.com

\begin{abstract}
Background \& Objective: Diabetes vascular complications are the leading cause of death in the world. Therefore, we investigated the effect of pyridoxal phosphate (PLP) on the formation of atheromatous plaque and renal function in atherosclerotic rats.

Materials \& Methods: Forty male Wistar rats were randomly divided into four groups of normal, atherosclerotic, and two similar groups under PLP treatment. Atherosclerosis was induced in rats by an atherogenic diet and all groups were treated with $0.18 \%$ of PLP in drinking water daily for three months. Hematoxylin and eosin stain was applied to assess the histopathological changes in the aorta of subjects. Insulin resistance index, the activity of the glyoxalase (GLO) system, lipid profile, low-density lipoprotein (LDL) oxidation markers, advanced oxidation protein products, and inflammatory markers, such as high-sensitivity C-reactive protein (hs$\mathrm{CRP}$ ) and TGF- $\beta 1$ were examined in all rats. In addition, serum creatinine levels and urinary protein excretion of all animals were measured.
\end{abstract}

Results: Atheromatous lesions were not observed in the aorta of PLP-treated atherosclerotic rats. Furthermore, PLP seemed to improve insulin function, lipid profile, kidney function parameters, GLO system activity, and inflammation. We found that PLP treatment decreased the formation of LDL oxidation products both in vitro and in vivo $(P<0.001)$.

Conclusion: According to our findings, PLP imposed a beneficial effect on vascular complications in atherosclerotic rats, which could be attributed to its antioxidant and anti-inflammatory properties. Moreover, PLP has positive impacts on insulin function, dyslipidemia, and GLO system activity.

Keywords: Atherosclerosis, Inflammation, Nephropathy, Oxidative stress, Pyridoxal phosphate Copyright $(\mathcal{C} 2021$, This is an original open-access article distributed under the terms of the Creative Commons Attribution-noncommercial 4.0 International License which permits
copy and redistribution of the material just in noncommercial usages with proper citation.

\section{Introduction}

Atherosclerosis and nephropathy are common vascular complications of diabetes and are considered the leading cause of death in the world $(1,2)$. According to the literature, oxidative stress and inflammation induce insulin resistance and vice versa (3). Furthermore, insulin resistance, even in the absence of hyperglycemia, is an important factor in the pathogenesis of vascular complications $(4,5)$. Insulin resistance evokes inflammation and dyslipidemia along with interfering with insulin signaling in vascular cells, which occurs during atherosclerosis progress (5). Moreover, oxidative stress increases the level of transforming growth factor- $\beta 1$ (TGF- $\beta 1$ ) in the kidney as an important factor in nephropathy (6).

Di-carbonyl compounds, such as methylglyoxal (MGO) have been known to lead to oxidative stress and inflammation contributing to a reduction in insulin secretion, insulin resistance, and vascular complications
$(7,8)$. The diminished activity of the glyoxalase (GLO) system, as the main defense against MGO accumulation, in the presence and absence of hyperglycemia plays role in insulin dysfunction, atherosclerosis (9), and nephropathy (10). Therefore, the induction of GLO system activity has recently become a major therapeutic target in vascular complications (11).

Preventive medicine is suggested to be the best approach for managing vascular complications (12). We recently investigated the favorable effect of some natural products on diabetic vascular complications (13-15). Pyridoxal phosphate (PLP), the active form of vitamin $\mathrm{B} 6$, is an essential coenzyme in numerous metabolic reactions (16) and might play a crucial role in protecting cells against oxidative stress. Some evidence exhibited that the deficiency of vitamin B6 along with variations in its metabolism contribute to vascular disorders due to the induction of endothelial dysfunction, oxidative stress, 
and inflammation $(16,17)$. Moreover, low circulating PLP is thought to be a possible indicator of inflammatory status and serum PLP is significantly correlated with the severity of coronary atherosclerosis (18).

With this background in mind, we investigated the impact of PLP on the formation of atheromatous plaque and renal function in atherosclerotic rats. Furthermore, the inhibitory effect of PLP on the generation of LDL oxidation products under in vivo and in vitro conditions was studied.

\section{Materials and Methods}

\section{Materials}

In this experimental study, all materials with analytical grade were purchased from Sigma and Merck Chemical Companies, Germany.

\section{In Vivo Studies}

Animals

Nine-week-old male Wistar rats weighing $185 \pm 15 \mathrm{~g}$ were purchased from Pasteur Institute of Iran, Karaj. Animals were housed under controlled conditions with free access to food and water. After two weeks, they were divided into the two main groups of normal and atherosclerotic. Atherosclerosis was induced in rats with an atherogenic diet. Afterwards, each of the two main groups was divided into two subgroups of 10 rats. The normal groups were fed a standard chow diet, while the atherosclerotic groups were fed an atherogenic diet (a chow diet containing 1\% cholesterol and $0.5 \%$ cholic acid).

The subgroups were named as "N" for the normal group and " $A$ " for the atherosclerotic group with no more treatments. The two similar subgroups under PLP treatment entailed N (PLP) and A (PLP), which received $0.18 \%$ PLP daily in their drinking water for three months. The experiment protocol was approved with the code of IR.ARUMS.REC.1397-176 by Animal Ethics Committee in accordance with the guidelines for the care and use of laboratory animals prepared by Ardabil University of medical sciences.

At the end of the intervention, fasting blood samples were collected from the hearts of the rats and were then transferred to test tubes with and without EDTA. Serum samples were obtained by $10 \mathrm{~min}$ centrifugation of blood at $1500 \mathrm{~g}\left(4^{\circ} \mathrm{C}\right)$ and were stored at $-70^{\circ} \mathrm{C}$ until further measurements. The aortas and kidneys of study rats were dissected and weighed immediately.

\section{Biochemical Parameters}

Fasting blood sugar (FBS), triglyceride (TG) (19), total cholesterol (TC), LDL, and high-density lipoprotein (HDL) were measured by enzymatic colorimetric methods. The LDL/HDL ratio was used to determine the atherogenic index. Serum insulin levels were determined using enzyme-linked immunosorbent assay (ELISA) using a rat insulin kit (Mercodia, Uppsala, Sweden). Moreover, a homeostasis model assessment of insulin resistance (HOMA-IR) was calculated.

\section{Methylglyoxal}

Serum MGO was measured utilizing reverse-phase high-performance liquid chromatography (HPLC) as explained previously (20).

\section{Oxidative and Inflammatory Markers}

Advanced oxidation protein products (AOPPs) as oxidative markers were assessed utilizing spectrophotometric detection according to the method of Witko-Sarsat et al. as described in our previous paper (20). Inflammatory markers, namely high-sensitivity Creactive protein (hs-CRP) and TGF- $\beta 1$ were measured using CUSABIO ELISA kits (Wuhan, Hubei, China).

\section{LDL Oxidation Products}

Conjugated dienes (CDs) in LDL lipids, as early products of LDL oxidation, were analyzed spectrometrically at $234 \mathrm{~nm}$. The fluorescence intensity of the end LDL oxidation product, named as oxidation fluorescent product (OFP), was recorded at the maximum emission of $430 \mathrm{~nm}$ upon excitation at $360 \mathrm{~nm}$ (20).

\section{Enzymatic Assay}

The activity of glyoxalase-1and 2 (Glo-I and Glo-II) in hemolysate was determined by measuring the rates of initial formation and hydrolysis of S-Dlactoylglutathione and enzymes activity (20).

\section{Pathologic Study}

The entire aortas of all rats from all groups were collected and fixed in $10 \%$ buffered formalin. Next, sections were prepared from each segment and were stained with hematoxylin and eosin (H\&E) stains for pathological examination.

\section{In Vitro Formation of LDL Oxidation Products LDL Extraction and Oxidation}

The LDL was extracted from serum specimens and was incubated with $\mathrm{CuSO}_{4}$ in the absence and presence of $0.18 \%$ PLP. The procedure of extraction and incubation was similar to what was explained previously (21). Briefly, LDL was isolated from the sera of normal rats with sodium heparin and was incubated in the presence and absence of $\mathrm{CuSO}_{4}(10 \mathrm{mmol} / \mathrm{L})$ and PLP (15 mmol/L).

\section{Oxidized LDL Products}

The resistance of LDL against oxidative modification with $\mathrm{CuSO}_{4}$, with and without PLP, was determined photometrically at $234 \mathrm{~nm}$ (22). In addition, the FOP of LDL was measured by the method explained in the "LDL Oxidation Products" section.

\section{Statistical Analysis}

Descriptive analysis of all data was expressed as mean \pm standard deviation (SD). Multivariate analysis of variance (MANOVA) test with Tukey posthoc test was used to compare the different variables assessed in all four groups utilizing the SPSS 16 (SPSS Inc., Chicago, IL., USA). Statistical significance was considered as $\mathrm{P}$-value $<0.05$. 


\section{Results}

\section{In Vivo Experiments}

The impacts of PLP administration on the formation of aortic lesions in normal and atherosclerotic rats are shown in Figures 1a-1b. Neointima (Figure 1b) and aorta plaque were found only in the atherosclerotic rats. However, the vitamin interfered with the generation of any aortic lesions. Furthermore, the histological observations in the aorta of the treated atherosclerotic rats were similar to those of the untreated and treated

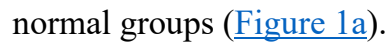
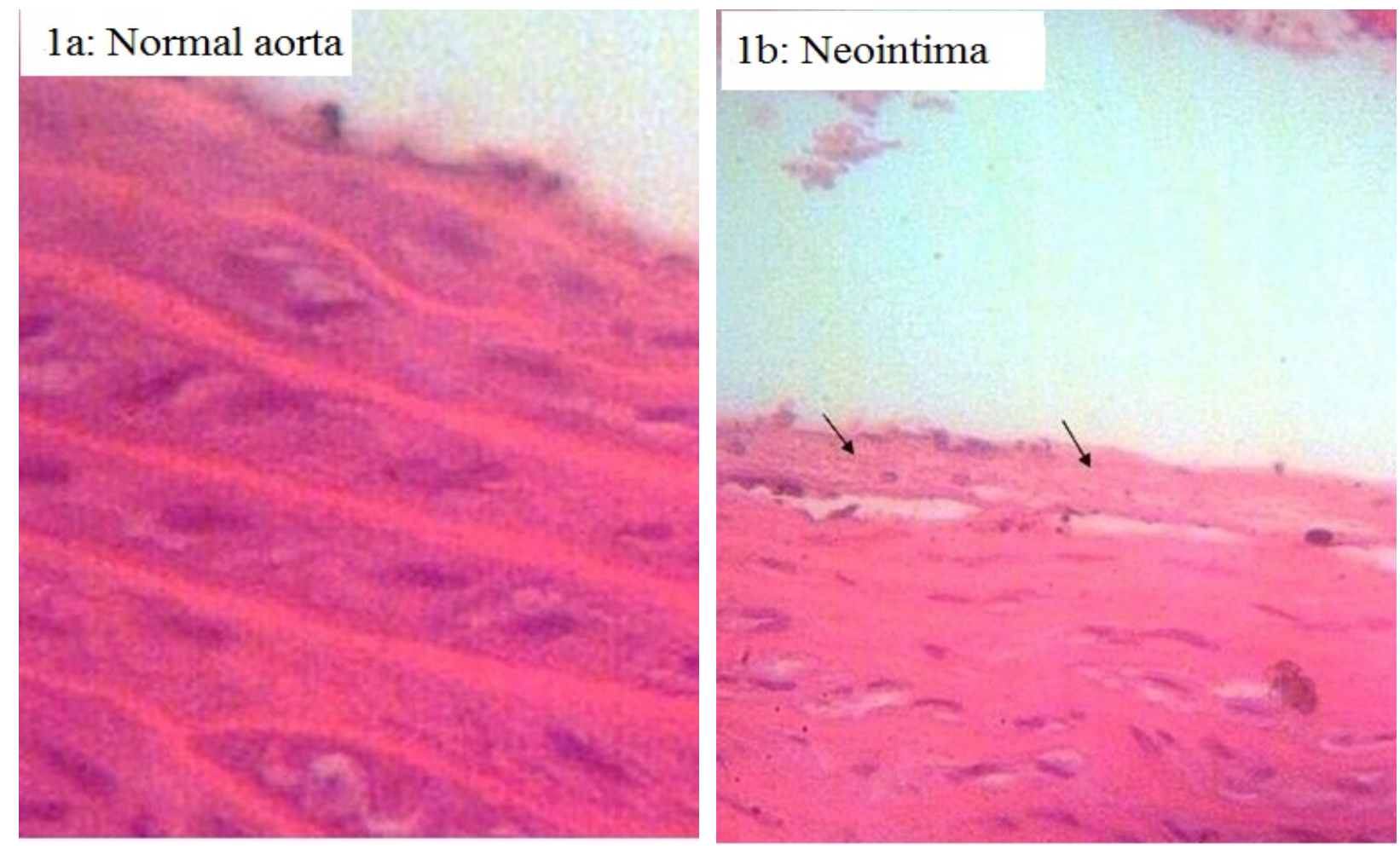

Figure 1. Effect of pyridoxal phosphate on atheromatous plaque formation in normal $(\mathrm{N})$ and atherosclerotic (A) rats (stained by H\&E \& original magnification $\times 200$ ). (a) Normal aorta in N, N (PLP) and A (PLP) groups. (b) Formation of neointima in A group. (c) Atherosclerosis plaque formation in A group

Table 1 represents the results of FBS, insulin, HOMAIR, lipid profile, and kidney dysfunction tests in all groups. Insulin, HOMA index (as a marker of insulin resistance), TG, TC, LDL, and the atherogenic index increased significantly due to atherosclerosis induction. Our findings demonstrated that PLP compensated for these changes and decreased the levels of $\mathrm{TC}$ and $\mathrm{TG}$ in the treated, normal, and atherosclerotic groups. Furthermore, $\mathrm{Cr}, \mathrm{PU}$, and kidney weight index (as kidney dysfunction tests), as well as TGF- $\beta 1$ (Table 2) were lower in the treated atherosclerotic group than in the untreated group $(P<0.001)$.

Results of comparing oxidative markers, inflammatory factors, the activity of the GLO system, and MGO levels between all groups are summarized in Table 2. Reductions in the AOPP, LDL oxidation products, and hs-CRP of the atherosclerotic rats reveals that the treatment exerted antioxidant and anti-inflammatory influences $(P<0.001)$. Furthermore, PLP induced GloI and Glo-II activity and diminished MGO levels in the mentioned groups $(P<0.001)$.

Table 1. The effect of pyridoxal phosphate (PLP) on FBS, insulin, HOMA-IR, lipid profile, and kidney function tests in normal $(\mathrm{N})$ and atherosclerotic $(\mathrm{A})$ rats.

\begin{tabular}{lcccc}
\multicolumn{1}{c}{ Parameter } & \multicolumn{3}{c}{ Groups } \\
\cline { 2 - 5 } & $\mathrm{N}$ & $\mathrm{N}(\mathrm{PLP})$ & $\mathrm{A}$ & $\mathrm{A}(\mathrm{PLP})$ \\
\hline Fasting blood sugar $(\mathrm{mmol} / \mathrm{L})$ & $4.49 \pm 0.26$ & $3.97 \mathrm{bb} \mathrm{b} \pm 0.29$ & $4.89 \pm 0.47^{*}$ & $4.66 \pm 0.4^{*}, \#$ \\
\hline Insulin $(\mu \mathrm{U} / \mathrm{mL})$ & $17.91 \pm 1.36$ & $18.53 \pm 0.67$ & $26.84 \pm 0.60^{*}$ & $21.89 \pm 0.55^{*}, \#$ \\
\hline HOMA-IR & $3.57 \pm 0.15$ & $3.26 \pm 0.17$ & $5.83 \pm 0.18^{*}$ & $4.53 \pm 0.16^{*}, \#$ \\
Triglyceride $(\mathrm{mmol} / \mathrm{L})$ & $0.93 \pm 0.03$ & $0.78 \pm 0.02^{*}$, & $2.83 \pm 0.08^{*}$ & $1.02 \pm 0.03^{*}, \#$ \\
\hline
\end{tabular}




\begin{tabular}{|c|c|c|c|c|}
\hline \multirow{2}{*}{ Parameter } & \multicolumn{4}{|c|}{ Groups } \\
\hline & $\mathrm{N}$ & N (PLP) & A & A (PLP) \\
\hline Total cholesterol $(\mathrm{mmol} / \mathrm{L})$ & $2.22 \pm 0.07$ & $1.99 \pm 0.09^{*}, \#$ & $6.42 \pm 0.16^{*}$ & $4.44 \pm 0.13 *, \#$ \\
\hline HDL (mmol/L) & $1.30 \pm 0.05$ & $1.25 \pm 0.05$ & $0.43 \pm 0.01 *$ & $0.78 \pm 0.02 *$ \\
\hline $\mathrm{LDL}(\mathrm{mmol} / \mathrm{L})$ & $0.43 \pm 0.02$ & $0.33 \pm 0.01$ & $4.68 \pm 0.12^{*}$ & $3.20 \pm 0.06 *$ \\
\hline $\mathrm{HDL} / \mathrm{LDL}$ & $0.33 \pm 0.01$ & $0.27 \pm 0.01 *$, \# & $11.00 \pm 0.21 *$ & $4.15 \pm 0.11 *$, \# \\
\hline Creatinine $(\mu \mathrm{mol} / \mathrm{L})$ & $61.50 \pm 4.24$ & $57.33 \pm 1.86$ & $96.50 \pm 5.74^{*}$ & $71.00 \pm 2.60^{*}, \#$ \\
\hline Proteinuria (mg/24 h) & $13.00 \pm 0.57$ & $10.87 \pm 0.40$ & $347.50 \pm 17.50^{*}$ & $170.00 \pm 8.11^{*}, \#$ \\
\hline Kidney weight index ( $\mathrm{g} / 100 \mathrm{~g}$ body weight) & $0.86 \pm 0.06$ & $0.87 \pm 0.05$ & $1.23 \pm 0.09^{*}$ & $0.98 \pm 0.08^{*}, \#$ \\
\hline
\end{tabular}

* Indicates significance of data comparing group $\mathrm{N}$ with other groups $(P<0.001)$

\# Indicates significance of data comparing group A with other groups $(P<0.001)$

Table 2. Effect of pyridoxal phosphate (PLP) on glyoxalase activity and methylglyoxal level as well as oxidative stress and inflammatory markers of the normal $(N)$ and atherosclerotic $(A)$ Rats.

\begin{tabular}{|c|c|c|c|c|}
\hline \multirow{2}{*}{ Parameter } & \multicolumn{4}{|c|}{ Groups } \\
\hline & $\mathbf{N}$ & $\mathbf{N}(\mathbf{P L P})$ & $\mathbf{A}$ & A (PLP) \\
\hline Methylglyoxal ( $\mu \mathrm{mol} / \mathrm{L})$ & $15.50 \pm 1.60$ & $13.12 \pm 0.63$ & $93.00 \pm 5.10^{*}$ & $48.60 \pm 2.30 *, \#$ \\
\hline Glyoxalase-I (U/mL) & $32.64 \pm 1.89 .90$ & $41.03 \pm 2.33^{*}, \#$ & $19.91 \pm 0.95^{*}$ & $30.75 \pm 2.01 *$ \\
\hline Glyoxalase-II (U/mL) & $33.42 \pm 1.96$ & $42.65 \pm 2.76^{*}$, \# & $21.53 \pm 1.20 *$ & $28.63 \pm 1.97^{*}, \#$ \\
\hline $\begin{array}{l}\text { Early oxidation products of LDL } \\
(\mu \mathrm{mol} / \mathrm{L})\end{array}$ & $13.81 \pm 0.89$ & $12.95 \pm 0.75^{*}, \#$ & $108.33 \pm 5.45^{*}$ & $67.61 \pm 5.67^{*}, \#$ \\
\hline $\begin{array}{l}\text { End oxidation products of LDL } \\
(\mu \mathrm{mol} / \mathrm{L})\end{array}$ & $248.73 \pm 12.33$ & $227.65 \pm 10.05^{*}, \#$ & $514.83 \pm 27.30^{*}$ & $432.37 \pm 22.97$ * \# \\
\hline $\begin{array}{l}\text { Advanced oxidation protein products } \\
(\mu \mathrm{mol} / \mathrm{L})\end{array}$ & $28.80 \pm 1.02$ & $18.82 \pm 0.85^{*}$ & $89.14 \pm 3.23 *$ & $61.83 \pm 2.84 *$, \# \\
\hline hs CRP (ng/mL) & $235.02 \pm 10.23$ & $187.15 \pm 7.55^{*}, \#$ & $596.77 \pm 25.21$ * & $400.23 \pm 17.81$, \# \\
\hline TGF- $\beta 1(\mathrm{pg} / \mathrm{mL})$ & $26.32 \pm 1.43$ & $23.89 \pm 1.09$ & $87.23 \pm 6.20^{*}$ & $56.46 \pm 3.62 *$ \\
\hline
\end{tabular}

${ }^{*}$ Indicates significance of data comparing group $\mathrm{N}$ with other groups $(P<0.001)$

\# Indicates significance of data comparing group A with other groups $(P<0.001)$

\section{In Vitro Study}

The levels of early and end LDL oxidation products (Table 3) decreased following the treatment.

Table 3. The effect of pyridoxal phosphate (PLP) on the formation of LDL oxidation products in the test tube experiment

\begin{tabular}{lcc}
\hline \multicolumn{1}{|c}{ Tube Content } & Early oxidation products $(\mu \mathrm{mol} / \mathrm{L})$ & End Oxidation products $(\mathrm{AU})$ \\
\hline $\mathrm{LDL}$ & $16.94 \pm 0.57$ & $15.17 \pm 0.44$ \\
\hline $\mathrm{LDL}+\mathrm{CuSO}_{4}$ & $135.56 \pm 4.55$ & $500.01 \pm 10.84$ \\
\hline $\mathrm{LDL}+\mathrm{CuSO}_{4}+\mathrm{PLP}$ & $50.83 \pm 1.22$ & $110.01 \pm 2.36$ \\
\hline
\end{tabular}

\section{Discussion}

According to the results of the current study, PLP reduced the levels of the markers of oxidative stress, carbonyl stress, and inflammation in atherosclerotic rats. It could be concluded that PLP prevented the formation of atheromatous lesions and improved kidney function in these animals. Furthermore, PLP had a corrective effect on insulin resistance, dyslipidemia, and the activity of the GLO system. Moreover, the inhibitory effect of the treatment on in vitro LDL oxidation confirms the obtained results.

The PLP was shown to impose positive effects on microvascular and macrovascular diseases. According to the pathologic investigation (Figures 1) and the 
results of kidney dysfunction tests (Table 1), this vitamin suppressed the development of atheromatous lesions and ameliorated kidney dysfunction by reducing serum TGF- $\beta 1$, creatinine, proteinuria, and kidney weight index in the atherosclerotic rats.

The studied vitamin diminished insulin resistance in the atherosclerotic rats (Table 1) owing to the beneficial impact on insulin function. Oxidative stress and inflammation might provoke insulin resistance and vascular complications. Di-carbonyl compounds, such as MGO, are sources of oxidative stress and inflammatory markers that reduce insulin secretion, insulin resistance, and vascular complications $(7,8)$. The activity of the GLO system, which is the most important pathway for decreasing methylglyoxal, is disturbed in atherosclerotic rats (Table 2). According to the literature, the effect of PLP on GLO system activity in atherosclerotic patients was not studied. Previously, the protective effect of PLP against STZinduced beta-cell dysfunction was reported (23).

The AOPPs are biomarkers of oxidative stress and inflammatory mediators and hs-CRP is a biomarker of systemic inflammation. These factors may enhance insulin resistance (24), atherosclerosis (25), and nephropathy (26). Based on Table 2, the treatment decreased the levels of AOPP and hs-CRP in normal and atherosclerotic rats $(P<0.001)$. The elevation of antioxidant enzyme activity in surgical patients under PLP treatment (27) confirms our results regarding the antioxidant effect of PLP.

Dyslipidemia is a prevalent aspect of atherosclerosis and nephropathy (28). Treatment with PLP corrected the lipid profiles of normal and atherosclerotic rats (Table 1). Moreover, the vitamin decreased the levels of TC, LDL, and atherogenic index in diabetic rats, whereas only TG and TC were reduced in the normal group $(P<0.001)$. As far as we know, the present investigation indicates the beneficial effect of PLP on the lipid profiles of atherosclerotic rats for the first time.

Hyperlipidemia and oxidative stress exacerbate LDL oxidation. Furthermore, hypercholesterolemia augments the changes in LDL contributing to the initiation and progression of atherosclerosis. Our results indicated that the treatment reduced the early and end oxidation products of LDL in normal and atherosclerotic rats in vitro (Tables 2 and 3 ). The diminishing effect of PLP on LDL oxidation in atherosclerotic rats had not been reported previously. Based on our previous studies, the treatments that inhibit the generation of the end oxidation products of LDL can prevent the development of all types of atheromatous lesions $(20,21)$.

\section{Conclusion}

Our findings revealed that PLP prevented the formation of atheromatous lesions and ameliorated renal dysfunction in atherosclerotic rats due to its antioxidant and anti-inflammatory properties. Furthermore, PLP had beneficial effects on insulin function, dyslipidemia, and GLO system activity. It could be concluded that PLP is useful in the treatment of diabetes.

\section{Acknowledgments}

The authors thank all those who helped them writing this article.

\section{Ethical considerations}

Ethical issues (Including plagiarism, informed consent, misconduct, data fabrication and/or falsification, double publication and/or submission, redundancy, etc.) have been completely observed by the authors.

\section{Funding and support}

Ardabil University of Medical Sciences financially supported this study.

\section{Conflict of Interest}

Authors declared no conflict of interest.

\section{Authors, contribution}

1) Authors who made substantial contributions to conception and design, and/or acquisition of data, and/or analysis and interpretation of data: Mahdavifard and Nakhjavani

2) Authors participated in drafting the article or revising it critically for important intellectual content: Mahdavifard

3) Author who finally approved the version to be submitted and revised the text: Mahdavifard \& Nakhjavani

\section{References}

1. Chilelli N, Burlina S, Lapolla A. AGEs, rather than hyperglycemia, are responsible for microvascular complications in diabetes: a "glycoxidationcentric" point of view. Nutr, Metab, Cardiovasc Dis. 2013;23(10):913-9. [DOI:10.1016/j.numecd.2013.04.004]

2. De Nicola LG, Liberti FB, Sagliocca ME, Conte A, Minutolo G. Sodium/glucose cotransporter 2 inhibitors and prevention of diabetic nephropathy: Targeting the renal tubule in diabetes. . Am $\mathrm{J}$ Kidney Dis. 2014;64 16-24. [DOI:10.1053/j.ajkd.2014.02.010]

3. Tangvarasittichai S. Oxidative stress and inflammation in diabetic complications. World $\mathrm{J}$ 
Diabetes.

$2015 ; 6(3): 456-80$

[DOI:10.4239/wjd.v6.i3.456]

4. Huang D, Refaat M, Mohammedi K, Jayyousi A, Al Suwaidi J, Abi Khalil C. Macrovascular complications in patients with diabetes and prediabetes. BioMed Res Int. 2017;2017: 7839101 [DOI:10.1155/2017/7839101]

5. Bornfeldt KE, Tabas I. Insulin resistance, hyperglycemia, and atherosclerosis. Cell Metab. 2011;14(5):575-85.

[DOI:10.1016/j.cmet.2011.07.015]

6. Kawanami D MK, Utsunomiya K. Dyslipidemia in diabetic nephropathy. Replace Ther 2016;2:16. [DOI:10.1186/s41100-016-0028-0]

7. Sarkar P, Kar K, Mondal MC, Chakraborty I, Kar M. Elevated level of carbonyl compounds correlates with insulin resistance in type 2 diabetes. Ann Acad Med Singapore. 2010;39(12):909-4.

8. Okura T, Ueta E, Nakamura R, et al. High serum advanced glycation end products are associated with decreased insulin secretion in patients with type 2 diabetes: A brief report. J Diabetes Res. 2017;2017:5139750.

[DOI:10.1155/2017/5139750]

9. Wortmann M, Peters AS, Hakimi M, Bockler D, Dihlmann S. Glyoxalase I (Glo1) and its metabolites in vascular disease. Biochem Soc Trans. 2014;42(2):528-33. [DOI:10.1042/BST20140003]

10. Dimitropoulos A, Rosado CJ, Thomas MC. Dicarbonyl-mediated AGEing and diabetic kidney disease. J Nephrol. 2020. [DOI:10.1007/s40620020-00718-z]

11. Nigro C, Leone A, Raciti GA, et al. Methylglyoxalglyoxalase 1 balance: The root of vascular damage. Int J Mol Sci. 2017;18(1):188-202. [DOI:10.3390/ijms18010188]

12. Nagai R, Shirakawa J, Fujiwara Y, et al. Detection of aGEs as markers for carbohydrate metabolism and protein denaturation. J Clin Biochem Nutr. 2014;55(1):1-6. [DOI:10.3164/jcbn.13-112]

13. Mahdavifard S NM. Effect of cysteine on transforming growth factor $\beta 1$ as the main cause of renal disorder in a rat model of diabetic nephropathy. J Mazandaran Univ Med Sci. 2019;29:95-101.

14. Mahdavifard S, Nakhjavani M. Effect of glutamine on oxidative stress, inflammatory, and glycation markers, and the activity of glyoxalase system in diabetic rats with atherosclerosis. J Mazandaran Univ Med Sci. 2019;28(170):33-42.

15. Mahdavifard S, Nakhjavani M. Effect of linalool on the activity of glyoxalase-I and diverse glycation products in rats with type 2 diabetes. J Mazandaran Univ Med Sci. 2020;30(186):24-33.
16. Nix WA, Zirwes R, Bangert V, et al. Vitamin B status in patients with type 2 diabetes mellitus with and without incipient nephropathy. Diabetes Res Clin Pract. 2015;107(1):157-65. [DOI:10.1016/j.diabres.2014.09.058]

17. Duncan A, Talwar D, McMillan DC, Stefanowicz F, O'Reilly DS. Quantitative data on the magnitude of the systemic inflammatory response and its effect on micronutrient status based on plasma measurements. Am J Clin Nutr. 2012;95(1):64-71. [DOI:10.3945/ajen.111.023812]

18. Ueland PM, McCann A, Midttun O, Ulvik A. Inflammation, vitamin B6 and related pathways. Mol Aspects Med. 2017;53:10-27. [DOI:10.1016/j.mam.2016.08.001]

19. Cole T, Kuisk I, Patsch W, Schonfeld G. Effects of high cholesterol diets on rat plasma lipoproteins and lipoprotein-cell interactions. $\mathrm{J}$ Lipid Res. 1984;25(6):593-603.

20. Mahdavifard S, Bathaie S, Nakhjavani M, Heidarzadeh H. L-cysteine is a potent inhibitor of protein glycation on both albumin and LDL, and prevents the diabetic complications in diabeticatherosclerotic rat. Food Res Int. 2014;62:909-16. [DOI:10.1016/i.foodres.2014.05.008]

21. Mahdavifard S, Bathaie SZ, Nakhjavani M, Taghikhani M. The synergistic effect of antiglycating agents (MB-92) on inhibition of protein glycation, misfolding and diabetic complications in diabetic-atherosclerotic rat. Eur $\mathrm{J}$ Med Chem. 2016;121:892-902. [DOI:10.1016/j.ejmech.2015.11.035]

22. Ravid M, Brosh D, Ravid-Safran D, Levy Z, Rachmani R. Main risk factors for nephropathy in type 2 diabetes mellitus are plasma cholesterol levels, mean blood pressure, and hyperglycemia. Arch Intern Med. 1998;158(9):998. [DOI:10.1001/archinte.158.9.998]

23. Kiran SG, Dorisetty RK, Umrani MR, et al. Pyridoxal 5' phosphate protects islets against streptozotocin-induced beta-cell dysfunction-in vitro and in vivo. Exp Biol Med. 2011;236(4):45665. [DOI:10.1258/ebm.2011.010361]

24. Bilgir O, Yavuz M, Bilgir F, et al. Relationship between insulin resistance, hs-CRP, and body fat and serum osteoprotegerin/RANKL in prediabetic patients. Minerva Endocrinol. 2018;43(1):19-26.

25. Swastini DA, Wiryanthini IAD, Ariastuti NLP, Muliantara A. Atherosclerosis prediction with high sensitivity C-reactive protein (hs-CRP) and related risk factor in patient with dyslipidemia. Macedonian J Med Sci. 2019;7(22):3887-90. [DOI:10.3889/oamjms.2019.526]

26. Sinha SK, Nicholas SB, Sung JH, et al. hs-CRP is associated with incident diabetic nephropathy: findings from the Jackson heart study. Diabetes 
Care. 2019;42(11):2083-9. [DOI:10.2337/dc182563]

27. Cheng CH, Huang SC, Chiang TY, Wong Y, Huang YC. Higher plasma pyridoxal phosphate is associated with increased antioxidant enzyme activities in critically Ill surgical patients. BioMed Res Int. 2013;2013:1-7. [DOI:10.1155/2013/572081]

\section{How to Cite This Article:}

Mahdavifard S, Nakhjavani M. Effect of Pyridoxal Phosphate on Atherosclerosis and Nephropathy Progression in Atherosclerotic Rats. J Adv Med Biomed Res. 2020; 29 (132) :21-27

\section{Download citation:}

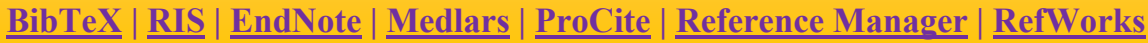

\section{Send citation to:}

63 Mendeley 2 Zotero ; RefWorks $\underline{\text { RefWorks }}$
28. Barter PJ. Lipoprotein metabolism and CKD: Overview. Clin Exp Nephrol. 2014;18:243-6. [DOI:10.1007/s10157-013-0866-9] 